Recepción: 20 / 04 / 2017

Aceptación: 20 / 05 / 2017

Publicación: 15 / 06 / 2017
Ciencias de la Educación

Artículo de Investigación

\title{
Aplicación de material educativo computarizado para cambiar las concepciones alternativas de cinemática en una dimensión
}
Educational material computer application to change the alternatives kinematic conception sin one dimension

\section{Material educacional, aplicação de computador para mudar as alternativas de concepção cinemática em uma dimensão}

Freddy A. Pereira-Guanuche ${ }^{\mathrm{I}}$

freddypereirag@hotmail.com

Fredis Franco-Pesantes II fredis20176@gmail.com

Kennya M. Pereira-Ruiz ${ }^{\text {III }}$ kennyita_music90@ hotmail.com

Kennya S. Ruiz-Veintimilla IV kennyaselene@gmail.com

Correspondencia: freddypereirag@hotmail.com

\footnotetext{
Magister en Enseñanza de la Física; Diploma Superior en Docencia Universitaria; Químico Industrial; Doctor en Química Industrial; Universidad Técnica de Machala, Machala, Ecuador.

Magister en Enseñanza de la Física ESPOL; Universidad Técnica de Machala, Machala, Ecuador.

Bioquímica Farmacéutica; Universidad Técnica de Machala, Machala, Ecuador.

Bioquímico Farmacéutico; Doctora en Bioquímica y Farmacia; Universidad Técnica de Machala, Machala, Ecuador.
} 


\section{Resumen}

En el transcurso de esta trayectoria académica, los estudiantes deben mejorar las habilidades necesarias para su vida personal y profesional; así como: la conceptualización, resolución de problemas, aprender individualmente, trabajar en equipo, pensamiento crítico en cualquier tema, entre otros; ya que están acostumbrados a recibir clases impartidas de manera tradicional, donde los estudiantes son entes pasivos receptores de la información. El propósito de este estudio fue de mejorar el rendimiento académico de los estudiantes en la unidad de Cinemática utilizando la elaboración de un material educativo computarizado. Se utilizó una muestra de 66 estudiantes registrados en un curso propedéutico de Física A y B de una universidad y fueron asignados a cuatro grupos intactos (A, B, C, y D). Al grupo A y B, se le aplicó el material educativo computarizado, después de la prueba de entrada con el mismo escenario pero diferente complejidad, en el grupo C y D, se desarrollaron la clase Tradicional basándose en los escenarios.

El 90,6 \% de los estudiantes se ubica en los niveles más bajos de conceptualización del concepto de campo (niveles 1, 2 y 3 definidos en este estudio), donde el nivel 3 corresponde a un reconocimiento y explicación parcial de significados del concepto. Este bajo nivel es concordante con bajos desempeños en las categorías de análisis, de operación (18,7 \%), expresión escrita (17,4 \%), representación $(14,1 \%)$ y resolución $(4,3 \%)$, de acuerdo con la teoría de Vergnaud, que afirma que un bajo nivel de conceptualización se caracteriza por bajos niveles de explicitación de invariantes y sus representaciones, con predominio de aspectos procedimentales de las operaciones sobre el uso de predicados de mayor riqueza conceptual.

Palabras claves: Rendimiento académico; escenario; concepto de términos de cinemática en una sola dimensión; aprendizaje de conceptos; campos conceptuales de Vergnaud; material educativo computarizado. 
Freddy A. Pereira-Guanuche; Fredis Franco-Pesantes; Kennya M. Pereira-Ruiz; Kennya S. Ruiz-Veintimilla

\begin{abstract}
During this academic journey, students must improve the skills necessary for personal and professional life, as well as the conceptualization, problem solving, individual learning, teamwork, critical thinking on any subject, among others, as are accustomed to traditionally taught classes where students are passive entitiesthat receive the information. The purpose of this study was toimprove the academic performance of students in the kinematicsunit using the development of a computerized educational material. A sample of 66 students enrolled in an introductory course in Physics A and B of a university and were assigned to four groups intact (A,B,C and D). Group A and $\mathrm{B}$, was applied to the computerized educational material, after the entrance test with the same scenario but different complexity in group $\mathrm{C}$ and $\mathrm{D}$, weredeveloped based on the traditional classroom scenarios.90.6\% of students were located at the lower levels of conceptualization of the concept of field (levels 1, 2 and 3 definedin this study), where the level 3 corresponds to a recognition and partial meanings of the concept. This low level is consistent with poor performance in the categories of analysis, operation (18.7\%), writing (17.4\%), presentation (14.1\%) and resolution (4.3\%), according Vergnaud theory, which states that a low level conceptualization is characterized by low levels of explicitin variants and representations, with prevalence of procedural aspects of the operations on the use of richer conceptuall predicates.
\end{abstract}

Key words: Academic performance; stage; kinematics conceptinterms ofa single dimension; concept learning; conceptual fieldsof Vergnaud; computerized educational material. 


\section{Resumo}

Durante esta jornada acadêmica, os alunos devem melhorar as habilidades necessárias para a vida pessoal e profissional, bem como a conceituação, resolução de problemas, aprendizagem individual, trabalho em equipe, pensamento crítico sobre qualquer assunto, entre outros, como estão acostumados a aulas tradicionalmente ensinadas onde os alunos são Entidades passivas que recebem a informação. O objetivo deste estudo foi melhorar o desempenho acadêmico dos alunos na unidade de cinemática usando o desenvolvimento de um material educacional informatizado. Uma amostra de 66 alunos matriculados em um curso introdutório em Física A e B de uma universidade e foram designados para quatro grupos intactos (A, B, C e D). Grupo A e B, foi aplicado ao material educacional informatizado, após o teste de entrada com o mesmo cenário, mas uma complexidade diferente nos grupos $\mathrm{C}$ e $\mathrm{D}$, foram desenvolvidos com base nos cenários de sala de aula tradicionais. 90,6\% dos alunos estavam localizados nos níveis mais baixos de conceituação Do conceito de campo (níveis 1, 2 e 3 definidos neste estudo), onde o nível 3 corresponde a um reconhecimento e significados parciais do conceito. Este baixo nível é consistente com o mau desempenho nas categorias de análise, operação $(18,7 \%)$, escrita $(17,4 \%)$, apresentação $(14,1 \%)$ e resolução $(4,3 \%)$, segundo a teoria de Vergnaud, que afirma que uma conceituação de baixo nível é Caracterizado por baixos níveis de variantes e representações explícitas, com prevalência de aspectos processuais das operações no uso de predicados conceituais mais ricos.

Palavras-chave: Performance acadêmica; etapa; Conceito de cinemática em termos de uma única dimensão; Aprendizagem de conceitos; Campos conceituais de Vergnaud; Material didático informatizado. 
Freddy A. Pereira-Guanuche; Fredis Franco-Pesantes; Kennya M. Pereira-Ruiz; Kennya S. Ruiz-Veintimilla

\section{Introducción}

En la Universidad pública ecuatoriana, el curso de admisión incluye Física entre las materias a aprobar. En el periodo lectivo 2014-2015 se realizó una experiencia piloto. Los mismos se incorporaron a una secuencia didáctica con la elaboración de material didáctico computarizado tutorial que contiene las diferentes concepciones alternativas y dificultades detectadas en cursos anteriores. Se evaluaron los aprendizajes con un pretesty postest, suministrados al inicio y a la finalización dela unidad académica.

La elaboración de Material Educativo Computarizado Tutorial de Cinemática (MECC) en una dimensión, es parte alternativa en el proceso de Aprendizaje-Enseñanza- para esta institución educativa, en la que recoge parte de la cinemática y dinámica como sustento fundamental de la mecánica; que estudia los objetos, y los conceptos afines de fuerza y energía, descripción de cómo se mueven los objetos y dinámica que trata de por qué se mueven los objetos y como lo hacen. Douglas Giancoli, (Pag 16).

Durante el transcurso del año escolar o semestre como se nombra en algunas instituciones de educación superior (IES) se ha podido observar un caso en particular; y es que la mayoría de los alumnos por no hablar en término general confunden la posición y el cambio de posición de un objeto. Resulta que, al pasar del tiempo se han realizado grandes investigaciones en el área de la física que han permitido que la comprensión de varios conceptos básicos de posición, tiempo, velocidad y aceleración sean captados por el estudiante de una manera menos compleja; sin embargo se debe admitir que se encuentran adheridas a diversas concepciones previas que interfieren en el proceso de aprendizaje de parte del estudiante. Las dificultades empiezan desde que el estudiante asimila las ideas del valor de la posición de un objeto en un espacio determinado y el 
cambio de posición en un intervalo de tiempo, la interpretación de lo que es velocidad y aceleración involucran sucesivos procesos de abstracción y comprensión de cocientes (Guidugli, S. y otros, 2004).

En la última década se observa un gran interés por parte del estudiante en conocer y adentrarse en el mundo de las tecnologías de la información y la comunicación (TIC) en donde se inserta un nuevo material educativo para los estudiantes de las nuevas generaciones, haciendo que los docentes en esta área analicen estos nuevos procesos educativos que mejoraran el desarrollo académico y una gran evolución en el proceso educativo que cursará el estudiante.

La reciente incorporación de estas tecnologías en todos los niveles de enseñanza ha planteado la necesidad de analizar de forma empírica la manera en que profesores y alumnos utilicen las TIC en el desarrollo del proceso educativo.

Las simulaciones y animaciones hacían un aporte en cuanto a la relación entre el movimiento sobre trayectorias rectilíneas y gráficos posición vs tiempo y también velocidad vs tiempo que se construyen simultáneamente con el movimiento sobre la trayectoria de algún objeto-imagen (pelota, personaje, auto). Se desarrollaron las actividades con el objetivo de generar conflictos cognitivos en los temas tratados.

El investigador no se conforma con la experimentación y las leyes obtenidas; quiere, además, buscar una explicación a los fenómenos observados y a las leyes descubiertas. Para ello comienza por establecer una serie de postulados o hipótesis. El método de enseñanza tiene argumentos sólidos que se rigen en conceptos comprobados por otros científicos. Sin duda fue el movimiento el primer aspecto del mundo físico que se estudió con detenimiento y su estudio puede remontarse hasta las antiguas civilizaciones del ASIA MENOR 
Freddy A. Pereira-Guanuche; Fredis Franco-Pesantes; Kennya M. Pereira-Ruiz; Kennya S. Ruiz-Veintimilla

Formulación del problema:

El propósito de este estudio es investigar las concepciones alternativas que tienen los estudiantes en cinemática en una dimensión utilizando la teoría de campos conceptuales de Vergnaud. Campo conceptual que Vergnaud considera, al conjunto de inconvenientes y ambientes que para ser analizados, necesita de conceptualizaciones, medios y graficaciones variadas, pero estrechamente correspondidas. En diversas actividades Vergnaud además de fine al campo conceptual; en primer lugar, como el conjunto de condiciones cuya destreza exhorta al dominio de conceptualizaciones del mismo origen o diferentes.

\section{Preguntas de investigación:}

El presente estudio trata de investigar como los estudiantes tienen diversas alternativas conceptuales de los términos básicos que se utilizan en la cinemática de una dimensión, para lo cual formulamos las siguientes preguntas.

¿Cómo afecta las concepciones alternativas de los términos básicos de Cinemática en una sola dimensión en el rendimiento de los estudiantes?

¿Cómo afecta las concepciones alternativas de los términos básicos en la resolución de problemas de cinemática en una sola dimensión?

\section{Objetivos de la investigación}

El presente estudio tiene como objetivos:

Desarrollar una estrategia cognoscitiva para mejorar las concepciones alternativas de los términos básicos de Cinemática en una sola dimensión en el rendimiento de los estudiantes. 
Diseñar y validar la elaboración de Material Educativo Computarizado de Cinemática en una dimensión que mejore el aprendizaje de los estudiantes.

Desarrollar habilidades de integración y conceptualización para mejorar el rendimiento académico en los estudiantes orientada a la resolución de problemas.

\section{Revisión de la literatura}

Una crítica común que se hace a la enseñanza de la Física en los cursos del nivel medio y la universidad, es que en ellos tratan temas demasiado modelados, llenos de restricciones y alejados de los problemas reales que son los que más interesan a los estudiantes.

\section{Cinemática}

La cinemática, rama de la mecánica que estudia la geometría del movimiento y describe este movimiento de los objetos sin interesarle las magnitudes que lo producen. Utiliza magnitudes fundamentales de longitud, posición, desplazamiento y tiempo como parámetros. La masa como magnitud física no interviene en este desarrollo, pero estas magnitudes están implícitas en los conceptos de velocidad y aceleración. [3]

Para determinar el movimiento delos cuerpos, se debe desarrollar en un sistema de referencia, en el que se determine un observador en el origen; quien hace el detalle de la situación.

\section{Creatividad y pedagogía}

La creatividad y la práctica de la pedagogía son dos procesos que deberían estar íntimamente relacionados, en donde la acción del profesor, como especialista en pedagogía, podría resultar necesaria y fundamental. Sin embargo, la creatividad a la vez que ofrece la posibilidad de desarrollo 
Freddy A. Pereira-Guanuche; Fredis Franco-Pesantes; Kennya M. Pereira-Ruiz; Kennya S. Ruiz-Veintimilla

al campo educativo y al docente, exige de éste una preparación acorde. Debe entender que enseñar no es sinónimo de transmitir cultura, sino de capacitar al estudiante para que por sí mismo la integre, recree y enriquezca. Si el docente quiere educar para el cambio y capacitar para la innovación debe hacerlo creativamente.

De la Torre [5] considera que la creatividad es una cualidad inherente a todo ser humano, está latente en casi todas las personas, es una cualidad sustantiva de las sociedades de empuje. La diversificación de criterios acerca de creatividad, crea limitaciones para asociar la investigación en ciencias y la pedagogía, sólo si los profesores de ciencias se disponen a proporcionar aportes integradores se podrá, en mejores condiciones orientar a los profesores en el desarrollo de actitudes creativas.

El docente debe despertar en el estudiante la curiosidad y estimular la creatividad de ellos, esto podría contribuir a modificar la actitud hacia el aprendizaje de las ciencias y la física. Si se inculca en los estudiantes el entusiasmo por descubrir por ellos mismos las leyes, principios y teoremas, tratando de fomentar la capacidad de asombro ante los fenómenos naturales y algoritmos matemáticos, a la vez que mantenemos la actitud de preguntarse el por qué de las cosas y la búsqueda sistemática de las respuestas, no estaríamos eliminando los conocimientos sino que éstos aparecen de una forma natural a lo largo del trabajo escolar.

González [6], afirma que en la enseñanza de la física se distinguen dos tendencias: (a) la física es una ciencia codificada, hecha, en la cual no hay nada que modificar y que está constituida por un conjunto de verdades inalterables descubiertas desde la antigüedad y (b) la física es una ciencia abierta que está en constante evolución y expansión, por ello su enseñanza debe permitir la reinvención de lo que es conocido por quien aprende, esto debe ser una condición necesaria aunque 
no suficiente para que el estudiante sea capaz de inventar o descubrir hechos matemáticos nuevos. De esta manera, cinemática en una sola dimensión será una parte que enfrente a los estudiantes con soluciones problemáticas que sean resueltas creando condiciones favorables al desarrollo de la creatividad.

Asimismo, se debe incentivar la utilización de estrategias de enseñanza de la cinemática en una sola dimensión que evidencien la presencia de actitudes creativas en los docentes. Actualmente el proceso de enseñanza de la cinemática en una sola dimensión está limitado por no ser creativo, este problema podría resolverse con la elaboración de programas de implantación de la creatividad, material educativo computarizado tutorial y medios informáticos en el aula.

\section{Estrategias coognositivas}

Basado en la literatura sobre ciertas corrientes que investigan estrategias para resolución de problemas y modelos propuestos por investigadores que se han dedicado a este tópico se puede citar a: Leer, Identificar, Dibujar y Evaluar. Con estos criterios tenemos ciertas estrategias cognoscitivas a aplicarse como:

a.- Leer atentamente y comprender el problema y tratar de que todos los términos empleados en el problema estén claros para darle un significado correcto de los mismos.

b.- Identificar las cantidades conocidas utilizando los símbolos y unidades apropiadas y colocar todas las unidades en el sistema compatible.

c.- Dibujar un esquema de la situación del problema, es decir haga un gráfico esquemático para visualizar el problema, en el mismo se debe de agregar los datos conocidos y desconocidos.- el 
Freddy A. Pereira-Guanuche; Fredis Franco-Pesantes; Kennya M. Pereira-Ruiz; Kennya S. Ruiz-Veintimilla

gráfico ayuda en el proceso de interacción entre el enunciado del problema y el estudiante mientras lee.

d.- Identificar los conceptos, leyes y principios que se deben de aplicar para la solución del problema.

e.- Sustituir los valores numéricos en la solución literal encontrada.

f.- Evaluar si la solución es físicamente aceptable, para esto se puede usarse un orden de magnitud y también sus unidades correspondientes. [7]

\section{Campo conceptual}

Vergnaud precisa al campo conceptual como un mecanismo de estudio para dar entendimiento a las dificultades estudiadas en la conceptualización de lo real; entendiendo la conceptualización como la propiedad del desarrollo cognitivo, también Vergnaud considera al campo conceptual, como el grupo de momentos cuya influencia necesita a su vez, el dominio de varios conceptos de naturaleza distinta; las estructuras multiplicativas, analizados como dificultades aditivas y múltiples, resolviéndolas únicamente con una multiplicación, una división o una combinación de esas operaciones.

Probablemente estos y otros campos conceptuales no son individualistas y unos pueden ser básicos para entender otros, pero aun así Vergnaud considera lucrativo el hablar de diferentes campos conceptuales. 


\section{Situaciones}

Son situaciones presentadas por Vergnaud, que dice que no es didáctica ya que unas son más difíciles y hay que analizarlas para que haya una relación en las tareas; por lo que, conocemos sus impedimentos del mismo, ya que hay gran diversidad de saber en los estudiantes que son modificados para así elegir las más acertadas. En los estudiantes hay gran diversidad de saber capaces de anticipar futuros eventos y generar nuevas conductas.

Existe una gran variedad de situaciones y los conocimientos de los alumnos son moldeados por las situaciones que encuentran y progresivamente dominan, particularmente por las primeras situaciones susceptibles de dar sentido a los conceptos y procedimientos que queremos que aprendan.

\section{Representaciones}

Sobre las teorías positivas y negativas, Vergnaud da a entender que una buena representación de las teorías plantadas en un escrito, nos permitirán a futuro gozar de un conocimiento valido o dañino.

\section{Esquemas}

A una estructura que no cambia el procedimiento para un establecido género de contextos, Vergnaud considera esquemas. Debe indagarse los conocimientos en ejercicio del sujeto, unidades cognitivas que permiten que las instrucciones del individuo sean prácticas. 
Freddy A. Pereira-Guanuche; Fredis Franco-Pesantes; Kennya M. Pereira-Ruiz; Kennya S. Ruiz-Veintimilla

Teóricamente los esquemas son los enlaces entre la conducta y la representación (1996 c, p.202). La conceptualización depende de la representación; que no es más que una relación entre los esquemas y las situaciones.

La coyuntura primordial entre la teoría y la práctica son los esquemas, que permiten la percepción, búsqueda y selección de conceptos disponibles para el sujeto y su comportamiento.

\section{Prueba de concepto}

Instrumento eficaz que mide los conocimientos en cinemática adquiridos por los alumnos, se consideró un test de 20 preguntas de opción múltiple, en la que una de las respuestas es correcta y las dos restantes son distractores.

Este test seleccionó preguntas del banco de preguntas sobre cinemática de una sola dimensión, contenido académico existente en la malla curricular de los estudiantes de bioquímica y farmacia. Una vez que tenemos el promedio de calificación que nos determina el conocimiento, e inmediatamente se realiza un análisis exhaustivo, cuyo objetivo primordial es una autoevaluación tanto para el catedrático como para los alumnos, este test se realizó al inicio del curso conocido como prueba de entrada, la que nos permite tener con claridad el nivel de conocimiento y otro test al termino del curso para determinar la ganancia de conocimiento.

\section{Explicación de propuesta: modelos y sistemas de planificación de materialeducativo computarizado (MECC).}

En la siguiente figura se resume la propuesta: 


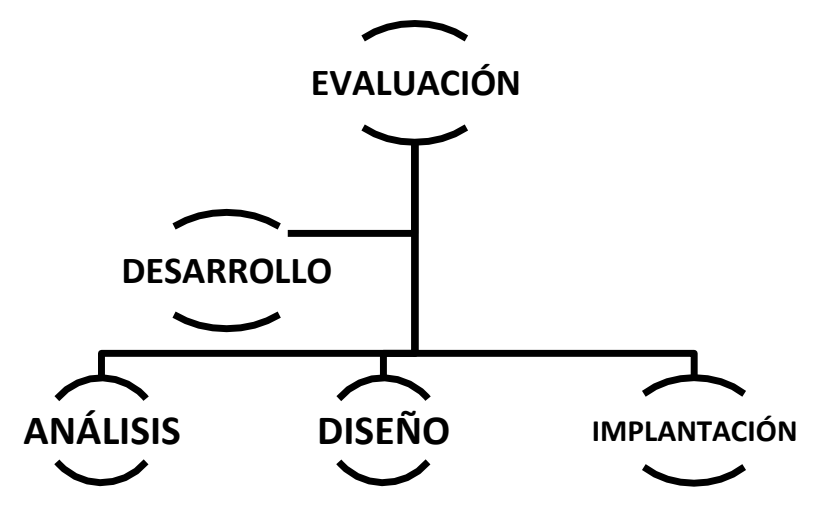

Figura $N^{\circ} 1 .-$ Fases del Diseño.

a. La fase de Análisis.-Define el problema, identifica la fuente del problema y establece alternativas de solución.

b. En la fase de Diseño.- Considera el producto de la fase de Análisis y planifica las posibles estrategias y se confecciona la instrucción.

c. En la fase de Desarrollo.- Se elabora las instrucciones y los materiales que se van a emplear.

d. En la fase de Implantación e Implementación.- Se socializa la instrucción.

e. En la fase de Evaluación.- Se evalúa en forma formativa y sumativa la instrucción.

f. Diseño de Medios Instruccionales.-En forma independiente se detalla la tecnología educativa a usar. 
Freddy A. Pereira-Guanuche; Fredis Franco-Pesantes; Kennya M. Pereira-Ruiz; Kennya S. Ruiz-Veintimilla

Metodología.

Sujetos

Para este estudio fueron 66 estudiantes de entre 17 a 21 años, del Primer de la Unidad Académica de Ciencias Químicas y de la Salud (UACQS) de la Universidad Técnica de Machala (UTMACH), en la asignatura de Física que aspiran a ser profesionales en la carrera de Bioquímica y Farmacia de esta institución educativa, los estudiantes no fueron colocados en forma aleatoria, divididos en cuatro grupos intactos homogéneos. El estudio se llevó a efecto en las aulas asignadas para este curso y tuvo una duración de 8 horas.

\section{Materiales}

Se utilizó la unidad instruccional de Cinemática, a la cual se le dedico 8 horas, la encuesta de conceptos generales de Cinemática, como pruebas de entrada y salida, las estrategias de resolución de problemas, al finalizar se administró la prueba de conocimientos para medir el rendimiento de los estudiantes.

\section{Resultados.}

Los resultados obtenidos que se presentan a continuación, se refieren a las características de las respuestas de los 66 estudiantes del preuniversitario de la UACQS de la Universidad Técnica de Machala.

\section{Resultados de la prueba conceptual de entrada y salida}

La Figura siguiente muestra en columnas la ganancia normalizada de la comparación de los promedios de test de entrada y salida. 


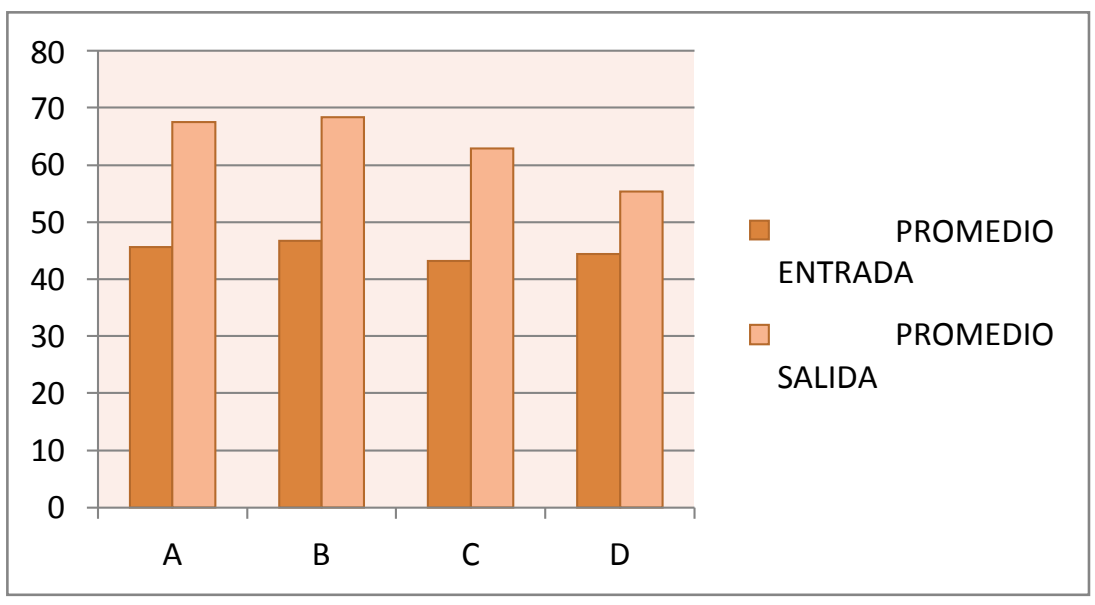

Figura $N^{\circ}$ 2.- Promedios de las pruebas de Entrada y Salida.

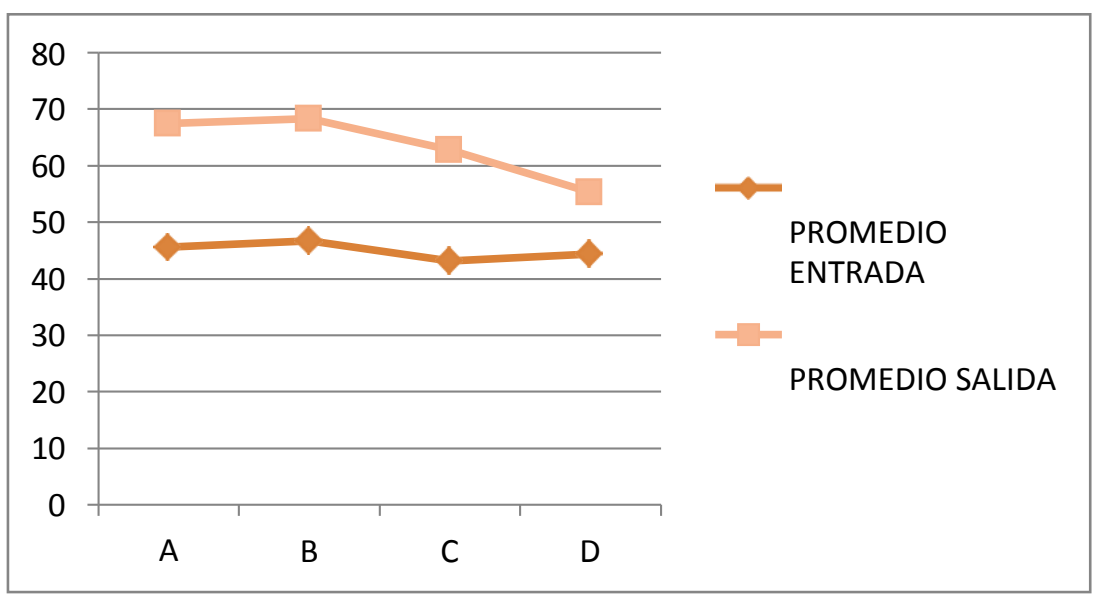

Figura 3.- Promedios en líneas de las pruebas de Entrada y Salida.

En la Figura 1 se observa con claridad los promedios de entrada y salida de la prueba de cinemática de los cuatro grupos intactos: grupo de entrada A (GEA), grupo de salida A(GSA),grupo de entrada B (GEB),grupo de salida B (GSB), grupo de entrada C (GEC), grupo de salida C (GSC), grupo de entrada D (GED), y grupo de salida D (GSD).

Así se demuestra estadísticamente los siguientes promedios: GEA 45.63, GSA 67.50; GEB 46.67 GSB, 68.33; GEC 43.13, GSC 62.81 y GED 44.41, GSD 55.29. 
Freddy A. Pereira-Guanuche; Fredis Franco-Pesantes; Kennya M. Pereira-Ruiz; Kennya S. Ruiz-Veintimilla

Se debe considerar que en las pruebas de entrada el grupo $\mathrm{C}$ es menor a los otros grupos, por lo que se debe mejorar el proceso de enseñanza-aprendizaje. En cambio que en la pruebas de salida el de mayor ganancia es el grupo A.

\begin{tabular}{|c|c|c|c|c|}
\hline $\begin{array}{c}\text { DATOS } \\
\text { ESTADÍSTICOS }\end{array}$ & A & B & C & D \\
\hline $\begin{array}{c}\text { GANANCIA } \\
\text { ABSOLUTA }\end{array}$ & 21,87 & 21,66 & 19.68 & 10,88 \\
\hline $\begin{array}{c}\text { GANANCIA } \\
\text { NORMALIZADA }\end{array}$ & 0,40 & 0,41 & 0,35 & 0,20 \\
\hline
\end{tabular}

Tabla $N^{\circ}$ 1.- Ganancia de los cuatro grupos.

\section{Discusión.}

En cuanto al análisis delos resultados de los promedios de entrada, en los cuatro grupos A, B, C y D, en nivel medio se ubican la mayoría de los estudiantes, identificando que los alumnos poseen dificultades en la comprensión; a pesar de la suficiente ilustración del catedrático, un grupo pequeño tiene un nivel de conceptualización correcto.

Examinando los promedios de las calificaciones del test de salida se identifica que los cuatro grupos tienen un aumento al comparar con el promedio de la prueba de entrada, señalando que los alumnos estudiantes lograr un conocimiento superior luego de la instrucción con el material educativo computarizado de cinemática (MECC), la estrategia de enseñanza aplicada y el aporte de la metodología tradicional.

Para determinar qué grupo tubo mayor adquisición de conocimientos luego de la instrucción, estadísticamente se estableció la ganancia normalizada de los promedios del test de entrada y de salida de cada de los cuatro grupos, estableciendo como consecuencia que los estudiantes del grupo B tiene mayor porcentaje de ganancia normalizada. 


\section{Conclusiones.}

El análisis de los resultados de los cuatro grupos señalaron el escaso nivel de conocimiento por parte de los alumnos al momento que se les aplico la pruebas de entrada y promedio alto en las pruebas de salida, lo que se debe entre otros factores anotados anteriormente, que el material educativo computarizado (MECC) tiene una excelente aportación en el proceso enseñanza de la cinemática en una dimensión.

\section{Bibliografía.}

[1] Giancoli Douglas (2006). "Principios con Aplicaciones de física" cuarta edición Prentice Hall.

[2] Alonso Marcelo y Finn Edward (1976). "Física: Fondo Educativo Interamericano".

[3]Alvarenga, Máximo (1997). Física General. Edit. Harla

[4] Tippens (1998). Física conceptos y aplicaciones. Edito. d.C. Graw Hill

[5] Castañeda Heriberto (2002). Física I. Ediciones Susaeta.

[6]Serway (2003). Física General. Vol. I

[7]Schaum, Daniel (1994). Teoría y problemas de física general. Edit. Mc Graw Hill

[8]Toro Á. Marcos (2006). Física Libro No2.Inst. Nacional Mejía

[9] Saquinaula, José; Lamilla Erick (2010) Guía práctica de Física Nivel Cero. Autores

[10] http://es.wikipedia.org/wiki/Cinem\%C3\%A1tica

[11] http://translate.google.com.ec/translate?hl=es\&langpair=en\%7Ces\&u=http://www.probertencyclopaedia. com/cgibin/res.pl\%3Fkeyword\%3DFall\%2Bof\%2BBodies\%26offset\%3D0

[12] http://translate.googleusercontent.com/translate_c?hl=es\&langpair=en\%7Ces\&u=http://physics.info/falli ng/\&rurl=translate.google.com.ec\&usg=ALkJrhiVlxf8r79DXkg06iopCg_VV_RjLQ

[13] Orhan, A. \&Ruhan, O. (2006). "The effects of Problem-Based Active Learning in Science Education on Students". Academic Achievement.

[14] Flores, J. (2006). "Modelo para el desarrollo del Aprendizaje basado en Problemas".

[15] Chacón, C. (2008). "Problemáticas fundamentales de la formación en fisica básica”. Bogotá: Tecné, Episteme y Didaxis. pág. 131-140.

[16] Manion \& L. Cohen, L., (1990). “to os inv sti aci n ucativa”. Espa a:

Editorial La Muralla, S.A. 
Freddy A. Pereira-Guanuche; Fredis Franco-Pesantes; Kennya M. Pereira-Ruiz; Kennya S. Ruiz-Veintimilla

[17] McDermott, L., \&Shaffer, P. (2001). “Tutorial s para Física intro uctoria”. Buenos Aires: Prentice Hall.

[18] De Landazábal, C., Bilbao, F., Otero, J., \& Caballero, C. (2002). "Formación inicial y rendimiento en física l prim r curso univ rsitario". Madrid: Revista de Educación.

[19] Pérez, G., B., M., Martínez Torregosa , J., \& C., S. (2005). ¿"Cómo promover el interés para la cultura ci ntífica"? Una propuesta fundamentada para la educación científica de jóvenes de 15 a 18 años. Santiago de Chile: Andros Impresores.

[20] O'Connor, J., \& Robertson, E. (2008). "Galileo Biography". Scotland: University of St Andrews.

$$
\text { Varignon, }
$$

Pierre

fr/archives/doc anciens/hmvol3502 pdf/p83 101 vol3502m.pdf

Fr/archives/doc_anciens/hmvol13502_pdf/p83_101_vol3502m.pdf "Du mouvement en general par toutessortes de courbes; $\&$ des forces centrales, tant centrifuges que centripetes, nécessaires aut corpsqui les décrivent" pp.

[22] Juan José Gómez Cárdenas (2007). “Cinemática relativista”, 2008

[23] Marcelo Alonso, Edward J. Finn (1976). “Física”. Fondo Educativo Interamericano.

[24] Richard Feynman (1974). Feynman lectures on Physics Volume 2 (en inglés). Addison Wesley Longman.

[25] Resnick Robert y Hlliday David (2004), “Física”, 4ta Edición, Volumen 1, CECSA, México.

[26]Universidad Autónoma Metropolitana Unidad Iztapalapa.

http://sgpwe.izt.uam.mx/Curso/209.Mecanica-Elemental-I/Tema/156.II-Cinematica-en-una-dimension.html. México D.F. 